\title{
Role of hydroxypropyl- $\beta$-cyclodextrin on freeze-dried and gamma-irradiated PLGA and PLGA-PEG diblock copolymer nanospheres for ophthalmic flurbiprofen delivery
}

This article was published in the following Dove Press journal:

International Journal of Nanomedicine

10 March 2012

Number of times this article has been viewed

\author{
Estefanía Vega' \\ M Antònia Egea' \\ Ana Cristina Calpena ${ }^{2}$ \\ Marta Espina' \\ M Luisa García! \\ 'Department of Physical Chemistry, \\ ${ }^{2}$ Department of Biopharmacy and \\ Pharmaceutical Technology, Institute \\ of Nanoscience and Nanotechnology, \\ Faculty of Pharmacy, University \\ of Barcelona, Barcelona, Spain
}

\begin{abstract}
Poly(D,L-lactide-co-glycolide) and poly(D,L-lactide-co-glycolide) with poly(ethylene glycol) nanospheres (NSs) incorporating flurbiprofen (FB) were freeze-dried with several cryoprotective agents and sterilized by $\gamma$-irradiation. Only when $5.0 \%(\mathrm{w} / \mathrm{v})$ hydroxypropyl- $\beta$-cyclodextrin (HP $\beta C D)$ was used, a complete resuspension by manual shaking and almost identical particle size of the NSs was obtained after freeze-drying. In vitro drug release and ex vivo corneal permeation of NSs with and without HP $\beta C D$ were evaluated. The presence of HP $\beta C D$ resulted in a reduction of burst effect, providing a more sustained release of the drug. A significant decrease in the FB transcorneal permeation of NSs containing HP $\beta C D$ was obtained, related to the slower diffusion of FB observed in the in vitro results. The uptake mechanism of the NSs was examined by confocal microscopy, suggesting that NSs penetrate corneal epithelium through a transcellular pathway. Ocular tolerance was assessed in vitro and in vivo by the Eytex ${ }^{\mathrm{TM}}$ and Draize test, respectively. Long-term stability studies revealed that $\gamma$-irradiated NSs stored as freeze-dried powders maintained their initial characteristics. Stability studies of the resuspended NSs after 3 months of storage in the aqueous form showed that NSs were stable at $4{ }^{\circ} \mathrm{C}$, while formulations stored at $25^{\circ} \mathrm{C}$ and $40^{\circ} \mathrm{C}$ increased their initial particle size.
\end{abstract}

Keywords: nanospheres, poly(D,L-lactide-co-glycolide), poly(ethylene glycol), hydroxypropyl$\beta$-cyclodextrin, freeze-drying, $\gamma$-irradiation

\section{Introduction}

Topical application of drugs into the eye is severely limited due to the rapid and extensive precorneal loss caused by drainage and high tear fluid turnover. This leads to a very short period of drug-cornea contact time, and less than $5 \%$ of the applied drug actually penetrates through the cornea and is able to reach intraocular tissues while a major fraction of the instilled dose is often absorbed systemically via the conjunctiva and nasolacrimal duct, producing undesirable side effects. ${ }^{1,2}$ Thus, the most frequently used dosage forms, solutions and suspensions, are compromised in their effectiveness.

A significant effort towards new drug-delivery systems to improve ocular administration has been observed in recent decades. ${ }^{3}$ Successful results have been obtained with polymeric colloidal nanospheres (NSs). Treatment with these systems increases bioavailability, reduces administration frequency, and promotes drug targeting to specific sites. ${ }^{4,5}$ Moreover, NSs have the advantage of offering a sustained
Correspondence: Estefanía Vega Freire Department of Physical Chemistry, Institute of Nanoscience and Nanotechnology, Faculty of Pharmacy, University of Barcelona, Av Joan XXIII s/n 08028 Barcelona, Spain

Tel +34934024552

$\mathrm{Fax}+34934035987$

Email evegafre7@far.ub.edu 
release of the drug entrapped in the polymeric matrix, so that the required tear levels and therapeutic effects can be achieved. ${ }^{6}$ Although a different number of polymers have been investigated for formulating biodegradable NSs, aliphatic polyesters such as poly(D-lactic acid) (PLA) and its copolymers with glycolic acid (PLGA) have been extensively used for controlled drug-delivery systems due to many favorable characteristics such as good biocompatibility, their ability to degrade into natural metabolites, and their safety profile for human use. ${ }^{7}$ Surface modification of PLGA NSs with polyethylene glycol (PEG) or PEGylation can modulate the interfacial properties of the system, and thus influence mucoadhesion to prolong the residence time of the particles in the precorneal area. ${ }^{8}$

The major obstacle that limits the use of such colloidal systems is their instability in aqueous mediums. Aggregation and fusion of particles are frequently noticed after a long period of storage of such systems. In order to improve the physical and chemical stability of these systems, the most commonly used process, which allows converting solutions or suspensions into solids of sufficient stability for distribution and storage is freeze-drying. ${ }^{9}$

Another limiting factor concerns sterilization, which is essential considering their ocular use. Commonly used sterilization techniques, such as steam or dry heat cannot be used for biodegradable aliphatic polyesters such as PLGA since they alter the physical and chemical properties of the polymer. Chemical sterilization with ethylene oxide causes serious toxicological problems due to residual content of the sterilizing agent. ${ }^{10}$ As PLGA is heat- and moisture-sensitive, $\gamma$-irradiation appears to be the only feasible alternative also offering the advantages of low chemical reactivity, low measurable residues, and fewer variables to control. ${ }^{11}$ A minimum absorbed dose of $25 \mathrm{kGy}$ is regarded as adequate for the purpose of sterilizing pharmaceutical products without providing any biological validation. ${ }^{12}$ Nevertheless, $\gamma$-irradiation induces dose-dependent chain scission and/or cross-linking and concomitant molecular weight (MW) loss of these polymers. The decrease in the polymer MW could have an effect in the release rate of the drug, depending on the active compound entrapped in the NSs. ${ }^{13-15}$

The incorporation of cyclodextrins (CDs) such as hydroxypropyl- $\beta$-cyclodextrin (HP $\beta C D$ ) into these polymeric drug-delivery systems can influence the mechanism by which drug is released. ${ }^{16,17} \mathrm{CDs}$ are water-soluble cyclic oligosaccharides, which can include a guest drug molecule in their hydrophobic cavity, allowing the solubility, stabilization, and transport of hydrophobic drugs. CDs offer an additional tool to modulate drug release rate from PLGA and PLGAPEG NSs.

Flurbiprofen (FB) was used as a model drug in this study. FB is a nonsteroidal anti-inflammatory drug, which has been introduced in ocular therapy not only for the management of inflammatory diseases concerning ocular structures, but also for use during eye surgery. ${ }^{18}$ Previous studies in our laboratory demonstrated successful incorporation of FB in PLGA NSs and physical stability in the form of suspension was determined. ${ }^{4}$

The main objective of this study was to evaluate the effect of freeze-drying with various cryoprotectants and the effect of $\gamma$-irradiation sterilization on the properties of FB-loaded PLGA and PLGA-PEG NSs. Different NSs with and without HP $\beta C D$ were obtained and in vitro release kinetics as well as corneal permeation studies were done to better understand the mechanism by which HP $\beta C D$ affects FB release rate.

\section{Material and methods \\ Materials}

PLGA 75:25 (Resomer ${ }^{\circledR}$ RG756S), PLGA 50:50 (Resomer ${ }^{\circledR}$ RG503), and PLGA 50:50-PEG Resomer ${ }^{\circledR}$ PEG types RGP d 5055, RGP d 50105, and RGP d 50155 (diblock 5, 10, and $15 \%$ PEG, respectively) were obtained from Boehringer Ingelheim (Ingelheim, Germany). Poloxamer 188 (P188, Lutrol ${ }^{\circledR}$ F68) was received from BASF (Barcelona, Spain). FB, D-(+)-monohydrated glucose, D-(+)-dihydrated trehalose, sucrose, HP $\beta C D$, and rhodamine-6G $(\mathrm{Rd})$ were obtained from Sigma (St Louis, MO). Double distilled water was used after filtration in a Millipore ${ }^{\circledR}$ (Millipore, Billerica, MA) system. All other chemicals and reagent used in the study were of analytical grade.

\section{Preparation of NSs}

Nanoparticles with a matrix structure (NSs) containing FB were prepared by the solvent displacement technique described by Fessi et $\mathrm{al}^{19}$ under the optimized conditions determined in our previous papers. ${ }^{4}$ Briefly, an organic solution of $90 \mathrm{mg}$ of polymer (PLGA or PLGA-PEG) in $25 \mathrm{~mL}$ of acetone containing $\mathrm{FB}(1.5 \mathrm{mg} / \mathrm{mL})$ was poured, while stirring moderately, into $50 \mathrm{~mL}$ of an aqueous $\mathrm{pH} 3.5$ solution containing $10 \mathrm{mg} / \mathrm{mL}$ P188. Acetone was then evaporated and the NS dispersion was concentrated to $10 \mathrm{~mL}$ under reduced pressure (B-480; Büchi, Flawil, Switzerland). Empty NSs were prepared using the same procedure but with no FB added to the organic phase. 


\section{Particle size and zeta potential}

The size of NSs was determined through dynamic light scattering (ZetaSizer Nano ZS; Malvern Instruments, Malvern, UK) and zeta potential was calculated from the electrophoretic mobility using the Helmholtz-Smoluchowski equation as reported previously. 4,5

\section{Entrapment efficiency}

The FB concentration in the NSs was determined by a modified reverse-phase high-performance liquid chromatography (RP-HPLC) method.$^{20}$ The amount of nonentrapped FB was separated by filtration/centrifugation technique using centrifugal filter devices Ultracel YM-100 (Amicon ${ }^{\circledR} ;$ Millipore) at $3000 \mathrm{rpm}$ for 12 minutes (Sigma 301K centrifuge; Sigma, Barcelona, Spain). Prior to filtration/centrifugation each sample was diluted with MilliQ (Millipore) water (1:20) to avoid deposition of free FB (possibly crystallized in the aqueous phase) on the NS surface and thus measured as encapsulated. The RP-HPLC system consisted of a Waters 1500 pump (Waters, Milford, MA) with a UV-Vis 2487 detector (Waters) set at $254 \mathrm{~nm}$. A reversed phase column (Spherisorb; Waters) ODS-2, Tracer (BC Aplicaciones Analíticas, Barcelona, Spain) with a flow rate of $1.5 \mathrm{~mL} /$ minute was used. The mobile phase consisted of acetonitrile/water (650:350) acidified with phosphoric acid ( $\mathrm{pH} 2.5)$.

The FB entrapment efficiency (EE) was calculated as indicated below:

$$
\text { EE }(\%)=\frac{\text { Total amount of FB }- \text { Free FB }}{\text { Total amount of FB }} \cdot 100
$$

\section{Freeze-drying of NSs}

FB-loaded NSs were freeze-dried without cryoprotectant and in the presence of $5 \%(\mathrm{w} / \mathrm{v})$ glucose, $5 \%(\mathrm{w} / \mathrm{v}) \mathrm{HP} \beta \mathrm{CD}$, $10 \%(\mathrm{w} / \mathrm{v})$ trehalose, $10 \%(\mathrm{w} / \mathrm{v})$ sucrose, and $5 \%(\mathrm{w} / \mathrm{v})$ trehalose plus $5 \%(\mathrm{w} / \mathrm{v})$ sucrose. The final concentration of the cryoprotectants used was chosen in order to maintain the tonicity of the ocular NS suspensions, which should be around $300 \mathrm{mOsm} / \mathrm{K} .{ }^{21}$ Osmolality was evaluated by freezing point measurements on a Fiske osmometer. The characteristics of the corneal surface and the lachrymal fluid $\mathrm{pH}$ as well as its buffer capacity are essential factors for ocular preparations since they may impact on the therapeutic effect of drugs and on their toxicity and stability. Tears have limited buffering capacity, which emphasizes the importance of the $\mathrm{pH}$ and buffer capacity of the instilled products..$^{22}$ In order to avoid these effects in the ocular assays presented here, test formulations were adjusted to tear fluid $\mathrm{pH}$ (7.4) using $0.1 \mathrm{M} \mathrm{NaOH}$.
A volume of $2 \mathrm{~mL}$ of the NS suspension were placed into glass vials and frozen by immersion in liquid nitrogen $\left(-196^{\circ} \mathrm{C}\right)$ and immediately freeze-dried for 24 hours at 0.05-0.03 mbar in a LyoQuest freeze-drier (Terrassa, Spain). Sample reconstitution was performed by adding $2 \mathrm{~mL}$ of MilliQ water to the dried cake followed by manual shaking. The macroscopic appearance and the particle size of the NSs after reconstitution were determined.

\section{Sterilization of NSs ( $\gamma$-irradiation)}

Freeze-dried NS powders (with and without FB) containing $5 \%(\mathrm{w} / \mathrm{v}) \mathrm{HP} \beta \mathrm{CD}$ as a cryoprotectant agent and the raw PLGA and PLGA-PEG copolymers were $\gamma$-irradiated using ${ }^{60} \mathrm{Co}$ as the irradiation source (Aragogamma, Barcelona, Spain) and received a dose of $25 \mathrm{kGy}$. According to the European Pharmacopoeia this dose is adequate for the purpose of sterilizing pharmaceutical products.

The influence of $\gamma$-irradiation on NS characteristics was evaluated by the study of particle size, zeta potential, and entrapment efficiency as described previously before and after irradiation.

\section{Differential scanning calorimetry (DSC)}

DSC analysis was performed on a Mettler Toledo DSC 823e System (Mettler Toledo, Barcelona, Spain). Samples (weight ranging from $2.65 \mathrm{mg}$ to $3.00 \mathrm{mg}$ ) were sealed in standard aluminium pans and heated at a rate of $10^{\circ} \mathrm{C} /$ minute over a temperature range of $0^{\circ} \mathrm{C}-140^{\circ} \mathrm{C}$. Indium (purity $\geq 99.95 \%$; Fluka, Buchs, Switzerland) was employed to check the calibration of the calorimetric system. The glass transition temperature $\left(\mathrm{T}_{\mathrm{g}}\right)$ of the polymer was obtained by taking the midpoint of the slope during glass transition. Samples were analyzed before and after their exposure to $\gamma$-irradiation.

\section{In vitro release}

In vitro release studies of FB from the NSs were carried out by the bulk equilibrium reverse dialysis bag technique. ${ }^{23}$ The experiments were performed under "sink conditions" to avoid the interference of the solubility of FB during the in vitro release. Briefly, $10 \mathrm{~mL}$ of each NS formulation was placed directly into $400 \mathrm{~mL}$ of isotonic phosphate-buffered saline (PBS) $0.1 \mathrm{M}, \mathrm{pH} 7.4$ at $32^{\circ} \mathrm{C}$ (ocular surface temperature) where numerous dialysis sacs (cellulose membrane MW cut off $12000 \mathrm{D}$, Iberlabo, Spain) containing $1 \mathrm{~mL}$ of isotonic PBS, had been previously immersed. The dialysis sacs were equilibrated with isotonic PBS prior to the experiments. At predetermined time intervals, a dialysis bag was withdrawn from the stirred release medium and simultaneously the PBS 
volume was replaced adding $1 \mathrm{~mL}$ of fresh buffer solution. The concentration of the released drug was assessed as described previously.

To evaluate the mechanism of release, data for the first $60 \%$ of the drug released were plotted in the KorsmeyerPeppas equation ${ }^{24}$ as $\log$ cumulative percentage of drug released versus log time, and the exponent $\mathrm{n}$ was calculated through the slope of the straight line. Where the drug transport mechanism from spherical matrices is by Fickian diffusion (case I transport) when $\mathrm{n} \leq 0.43$, if $0.43<\mathrm{n}<0.85$, it indicated anomalous (non-Fickian) transport and for values of $\mathrm{n} \geq 0.85$, case II or zero order release kinetics was indicated. Occasionally, values of $\mathrm{n} \geq 0.85$ for release from spheres have been observed, which has been regarded as super case II kinetics.

The release profile of FB was tested after NSs sterilization. The similarity factor $\mathrm{f}_{2}$ was calculated from the mean release data before and after $\gamma$-irradiation. ${ }^{25}$

\section{Ex vivo study}

These studies used white New Zealand rabbits weighing $1.8 \mathrm{~kg}$ to $2.2 \mathrm{~kg}$ free of any signs of ocular inflammation or gross abnormalities. All experiments were performed according to the Association for Research in Vision and Ophthalmology resolution for the use of animals in research and were approved by the local ethics committees for animal experimentation.

\section{Confocal laser scanning microscopy (CLSM)}

In order to evaluate the uptake mechanism of the NSs through the corneal epithelium, fluorescent NSs incorporating Rd as a fluorescent marker were prepared. Fifty micrograms of Rd was added to the acetone solution and the NSs were prepared as described previously.

Entrapment efficiency was calculated from the amount of the free Rd present in the aqueous phase of the formulations (obtained by filtration/centrifugation at $3000 \mathrm{rpm}$ for 12 minutes) measuring the amount of $\mathrm{Rd}$ by fluorescence spectroscopy $\left(\lambda_{\mathrm{ex}}=523 \mathrm{~nm}, \lambda_{\mathrm{em}}=548 \mathrm{~nm}\right)$.

In vitro release experiments were performed to confirm that the fluorescent marker remained associated with the particles during the whole experiment. A bulk equilibrium reverse dialysis bag technique was performed (see In vitro release section). After 2 hours of incubation, the corresponding dialysis bags were withdrawn and the $\mathrm{Rd}$ concentration was determined by fluorescence spectroscopy $\left(\lambda_{\mathrm{ex}}=523 \mathrm{~nm}, \lambda_{\mathrm{em}}=548 \mathrm{~nm}\right)$.
A volume of $50 \mu \mathrm{L}$ of each formulation was administered to the cul-de-sac of fully awake conscious rabbits. At 2 hours postadministration, rabbits were sacrificed and freshly excised corneal specimens were directly mounted, epithelial side up, on a glass slide and examined by CLSM without further tissue processing.

Confocal images were taken using an Olympus Fluoview 500 confocal microscope adapted to an inverted IX70 microscope (Serveis Cientificotècnics; University of Barcelona, Barcelona, Spain). Samples were excited with an argon ion laser at $488 \mathrm{~nm}$ wavelength.

\section{Corneal permeation studies}

Rabbits were sacrificed by intravenous overdose of sodium pentobarbital and the corneas, with a $2 \mathrm{~mm}$ ring of sclera, were immediately excised and mounted in a perfusion apparatus maintained at $37^{\circ} \mathrm{C}$. Glutathione bicarbonate Ringer's solution was added to the epithelial $(1 \mathrm{~mL})$ and the endothelial $(3.2 \mathrm{~mL})$ compartment. To ensure oxygenation and agitation, an $\mathrm{O}_{2}-\mathrm{CO}_{2}(95: 5)$ mixture was aerated through each compartment. Samples from the endothelial side were withdrawn at different times and replaced with fresh glutathione bicarbonate Ringer's solution. All analyses were carried out by the RP-HPLC method previously described.

Each experiment was repeated six times. The apparent corneal permeability coefficient $\left(\mathrm{P}_{\text {app }}\right)$ was determined using the expression:

$$
\mathrm{P}_{\mathrm{app}}=\frac{1}{\mathrm{~A} \cdot \mathrm{C}_{\mathrm{o}} \cdot 60} \frac{\Delta \mathrm{Q}}{\Delta \mathrm{t}}
$$

where $\mathrm{Q}$ is the total amount permeated at time $\mathrm{t}, \Delta \mathrm{Q} / \Delta \mathrm{t}$ is the steady-state slope of the linear portion of the plot for the amount of drug in the receiving chamber $(\mathrm{Q})$ versus time $(\mathrm{t})$, $A$ is the exposed corneal surface $\left(0.64 \mathrm{~cm}^{2}\right), C_{0}$ is the initial concentration of drug in the donor cell, and 60 represents the conversion of minutes to seconds.

\section{Determination of corneal hydration levels}

Corneal hydration levels were investigated by measuring total water content by desiccation (gravimetric method). Each corneal sample was carefully removed from the scleral ring and weighed $\left(\mathrm{W}_{\mathrm{W}}\right)$. It was then desiccated at $100^{\circ} \mathrm{C}$ for 6 hours to determine dry corneal weight $\left(\mathrm{W}_{\mathrm{d}}\right)$. The corneal hydration level $(\%)$, defined as $\left[1-\left(\mathrm{W}_{\mathrm{w}} / \mathrm{W}_{\mathrm{d}}\right)\right] \times 100$, was determined both on untreated corneas and corneas recovered from the permeation tests. 


\section{Ocular tolerance analysis}

In vitro Eytex ${ }^{\mathrm{TM}}$ test

In view of the 3R's concept (reduction, refinement, and replacement), many alternative techniques such as the Eytex $^{\mathrm{TM}}$ test are already available to reduce and replace animal testing. In vitro screening will serve as a preliminary method to assess possible risk in animal studies. The Eytex ${ }^{\mathrm{TM}}$ system (In Vitro International, Irvine, CA) is an in vitro method for predicting in vivo ocular irritation that uses a vegetable protein extracted from jack beans. ${ }^{26}$ Like the cornea of the eye, this clear protein gel becomes cloudy when it comes into contact with an irritant. The molecules in the Eytex ${ }^{\mathrm{TM}}$ protein gel also have a highly organized structure, which is changed by irritants. The more irritant a substance is, the more the structure of the molecule groups is changed and the cloudier the gel appears. The changes in protein structure induced by the test product may be quantified by measuring the resulting changes in turbidity. Comparison of these optical density measurements to those produced by standard chemical irritants allows calculation of an "irritancy score" called "irritection draize equivalent" (IDE), that has been shown to be directly related to the potential corneal irritancy of the sample. ${ }^{27}$ According to the IDE score, the irritancy potential of the formulations may be classified as "minimal" (0.0-12.5), "mild" (12.5-30.0), "moderate" (30.0-51.0), or "severe" (>51.0). The analysis of the data and the IDE calculations were performed by Tecan ${ }^{\circledR}$ software (In Vitro International).

\section{In vivo Draize test}

Since Eytex ${ }^{\mathrm{TM}}$ is not accepted as an alternative method by regulatory authorities, ${ }^{28}$ the in vivo Draize test was performed to confirm the results obtained for the in vitro irritancy test. In vivo eye irritation was assessed in New Zealand white rabbits by the Draize eye test, which is the official technique for the evaluation of cosmetic and pharmaceutical products for ocular instillation by the interpretation of Kay and Calandra. ${ }^{29,30}$ A single instillation of $50 \mu \mathrm{L}$ of each sample was administered in one eye, using the untreated contralateral eye as a control. Readings were performed at 1 hour after sample application, then at 1, 2, 3, 4, and 7 days, respectively. The method provided an overall scoring system for grading the severity of ocular lesions involving the cornea (opacity), iris (inflammation degree), and conjunctiva (congestion, swelling, and discharge). The Draize score was determined by visual assessment of changes in these ocular structures. The mean total score (MTS) was calculated using the following equation:

$$
\operatorname{MTS}=\sum \frac{\mathrm{x}_{1}(\mathrm{n})}{5}+\sum \frac{\mathrm{x}_{2}(\mathrm{n})}{2}-\sum \frac{\mathrm{x}_{3}(\mathrm{n})}{5}
$$

where $x_{1}(n), x_{2}(n)$, and $x_{3}(n)$ are the cornea, conjunctiva, and iris scores, respectively, and $n$ being the number of rabbits included in the ocular tolerance assay. Three animals were used for each product, in accordance with the principles of the 3R's (reduction, refinement, and replacement). ${ }^{31}$

\section{Storage conditions during stability studies}

For long term experiments, samples of NS powders freezedried and $\gamma$-irradiated, were stored at $4^{\circ} \mathrm{C}, 25^{\circ} \mathrm{C}$, and $40^{\circ} \mathrm{C}$ protected from light. NS formulations were resuspended immediately and after 6 months of storage in $2 \mathrm{~mL}$ of isotonic PBS $0.1 \mathrm{M}, \mathrm{pH} 7.4$ in order to maintain the $\mathrm{pH}$ and the tonicity of the samples. The morphometrical properties as well as entrapment efficiency, $\mathrm{pH}$, and tonicity of the resuspended formulations were evaluated after 1 and 3 months of storage at $4^{\circ} \mathrm{C}, 25^{\circ} \mathrm{C}$, and $40^{\circ} \mathrm{C}$ protected from light.

\section{Statistical analysis}

Values reported are mean \pm standard deviation (SD). Mean values of the data obtained were compared among groups using one-way analysis of variance and Student's $t$-test for two-group comparison. Two-way analysis of variance was used to determine the effect of temperature, storage time, and the interaction between these factors in the long-term stability studies. Differences were considered significant at the $P<0.05$ level.

\section{Results and discussion NSs characterization}

FB-loaded NSs were prepared in order to evaluate the effect of the different copolymers on particle size, zeta potential, and entrapment efficiency. All formulations prepared showed suitable particle size for ocular administration lower than $250 \mathrm{~nm} .{ }^{32}$ The polydispersity index was lower than 0.1 indicating narrow particle size distribution.

As can be seen in Table 1, NSs prepared from different copolymer compositions, PLGA (50:50 and 75:25) did not show any significant difference in particle size for blank NSs. Nevertheless, particle size increased for drug-loaded NSs prepared with the copolymer PLGA 75:25. This can be explained by the high ratio of lactic acid in the 75:25 PLGA, which are more hydrophobic than the glycolic acid units, increasing the hydrophobic character of the copolymer, which has more affinity for the drug, resulting in a higher entrapment efficiency as well as higher particle size. 
Table I Physicochemical properties and entrapment efficiency of PLGA and PLGA-PEG NSs with and without FB

\begin{tabular}{llllll}
\hline NS composition & MW (KDa) & Size \pm SD $(\mathbf{n m})$ & PI \pm SD & $\zeta$-potential \pm SD (mV) & EE \pm SD (\%) \\
\hline PLGA 75:25 & 98 & $202.0 \pm 1.8$ & $0.076 \pm 0.009$ & $-25.7 \pm 1.3$ & - \\
PLGA 75:25 + FB & & $232.8 \pm 1.9$ & $0.048 \pm 0.045$ & $-25.8 \pm 1.2$ & $94.6 \pm 0.4$ \\
PLGA 50:50 & 34 & $205.3 \pm 1.4$ & $0.051 \pm 0.003$ & $-34.0 \pm 0,5$ & - \\
PLGA 50:50 + FB & & $197.5 \pm 1.4$ & $0.062 \pm 0.022$ & $-33.5 \pm 0.4$ & $90.3 \pm 3.2$ \\
PLGA 50:50-PEG 5\% & 95 PLGA + 5 PEG & $146.8 \pm 2.1$ & $0.081 \pm 0.014$ & $-22.5 \pm 0.3$ & - \\
PLGA 50:50-PEG 5\% + FB & & $147.7 \pm 1.8$ & $0.052 \pm 0.043$ & $-22.2 \pm 0.2$ & $87.7 \pm 3.7$ \\
PLGA 50:50-PEG 10\% & \multirow{2}{*}{ 45 PLGA + 5 PEG } & $121.3 \pm 0.9$ & $0.090 \pm 0.002$ & $-17.3 \pm 0.4$ & - \\
PLGA 50:50-PEG 10\% + FB & & $123.3 \pm 1.6$ & $0.094 \pm 0.063$ & $-17.0 \pm 0.6$ & $80.5 \pm 5.0$ \\
PLGA 50:50-PEG 15\% & 28 PLGA + 5 PEG & $90.2 \pm 0.9$ & $0.094 \pm 0.021$ & $-12.8 \pm 0.2$ & - \\
PLGA 50:50-PEG 15\% + FB & & $96.8 \pm 0.8$ & $0.085 \pm 0.019$ & $-12.3 \pm 1.7$ & $75.0 \pm 2.4$ \\
\hline
\end{tabular}

Note: Mean \pm SD, $\mathrm{n}=3$.

Abbreviations: EE, entrapment efficiency; FB, flurbiprofen; MW, molecular weight of PLGA and PLGA-PEG copolymers used for NS preparations; NSs, nanospheres; PI, polydispersity index; PLGA, Poly(D,L-lactide-co-glycolide); PLGA-PEG, poly(D,L-lactide-co-glycolide) with poly(ethylene glycol); SD, standard deviation; $\zeta$-potential, zeta potential.

Particle size obtained was significantly lower as the content of PEG in the copolymer increased. This could be largely due to the reduction in MW and increase in amphiphilic character of the copolymer with increasing PEG composition. Thus, the combined effect of MW and amphiphilicity largely contributed to the reduction in particle size..$^{33}$

The high entrapment efficiency obtained (75\%-90\%) for both PLGA and PLGA-PEG NSs could be explained by the hydrophobic nature of FB. The presence of hydrophilic PEG segments resulted in lower entrapment efficiency values in comparison with PLGA NSs. FB was incorporated into NSs made from PLGA-PEG diblock copolymers with a fixed PEG block (5 KDa) and varying PLGA segments (28-95 KDa; see Table 1). Higher entrapment efficiency values were obtained for PLGA-PEG NSs as the MW of the PLGA block was increased due to their bigger size, which result in a smaller surface area for drug loss as well as a larger core for an increased drug entrapment. ${ }^{34}$

The results from the zeta potential show highly negative values for PLGA NSs due to the ionization of carboxylic end groups of polymer on the surface, whereas PLGA-PEG NSs had lower zeta potential values due to the presence of PEG on their surface which covers the surface charges..$^{35}$

PLGA 50:50 showed a large negative zeta potential value in comparison with PLGA 75:25. It has been described that the presence of $\mathrm{P} 188$, the emulsifier used during the preparation of NSs, can contribute to a reduction in their surface charge.$^{36}$ In fact, it is known that this emulsifier tends to bind to the NS surface through hydrophobic interactions involving its polyoxypropylene chains, while the hydrophilic polyoxyethylene chains protrude into the surrounding medium, thus masking the negative surface charges present in the NSs. In the light of these considerations, it is possible that a stronger interaction is established between P188 and the PLGA 75:25 NSs (which has a more hydrophobic surface due to the higher proportion of lactic acid/glycolic acid), as compared to the other copolymer used, thus justifying the lower values of zeta potential observed.

\section{Influence of cryoprotectant on freeze-drying}

PLGA and PLGA-PEG NSs were freeze-dried in the presence of a monosaccharide $(5 \%(\mathrm{w} / \mathrm{v})$ glucose $)$, two disaccharides $(10 \%(\mathrm{w} / \mathrm{v})$ sucrose, $10 \%(\mathrm{w} / \mathrm{v})$ trehalose $)$, a combination of $5 \%(\mathrm{w} / \mathrm{v})$ sucrose plus $5 \%(\mathrm{w} / \mathrm{v})$ trehalose, and a cyclic oligosaccharide (5\% (w/v) HP $\beta C D)$. Carbohydrates are widely used for the protection of nanoparticles during freeze-drying. ${ }^{9}$ Adding cryoprotectants to the formulations resulted in no significant change in morphometrical properties (data not shown), except for the formulations containing $5 \%(\mathrm{w} / \mathrm{v}) \mathrm{HP} \beta \mathrm{CD}$, where a slight increase in particle size and drug loading and a decrease in zeta potential values was determined, which indicate the presence of $\mathrm{HP} \beta C D$ at the surface of the particles. ${ }^{37}$

As can be observed in Figure 1, when PLGA and PLGAPEG NSs were freeze-dried without cryoprotectant, full redispersion of the particles could not be achieved after rehydration, even using sonication, indicating a strong type of aggregation. From the results obtained, we can conclude that $\mathrm{P} 188$ used as stabilizer in the preparations of NSs did not protect formulations during freeze-drying. This aggregation is explained by an increase in the solubility of P188 in the bulk solution during the freezing process. The solubility of poloxamers is higher in cold than in hot water, due to the hydrogen bond formation between the water molecules and the numerous ether type oxygen bonds of the poloxamers. A decrease in temperature favors the solvation of the poloxamer by increasing hydration 


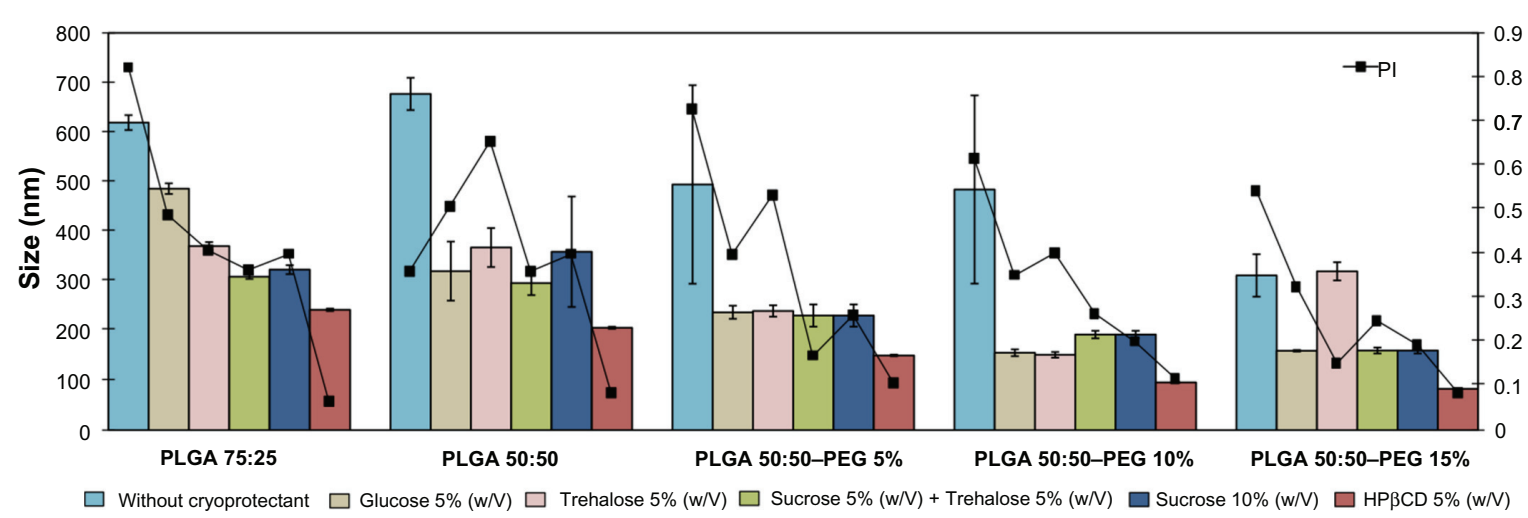

Figure I Particle size and PI of reconstituted FB-loaded NSs in the presence of different cryoprotectants.

Note: Error bars represent \pm SD.

Abbreviations: FB, flurbiprofen; HPßCD, hydroxypropyl- $\beta$-cyclodextrin; NS, nanospheres; PI, polydispersity index; PLGA, Poly(D,L-lactide-co-glycolide); PLGA-PEG, poly(D,L-lactide-co-glycolide) with poly(ethylene glycol); SD, standard deviation.

of the polyethylene oxide shell and polypropylene blocks, thus the dynamic motion of the surface attached chains is broken and the latter tend to remain in the bulk solution. ${ }^{38}$

As shown in Figure 1, when glucose, trehalose, sucrose, and a combination of trehalose and sucrose were used, NSs resuspension was achieved using sonication, but particle size was increased and trace amounts of aggregates were observed, although small in comparison with NSs without cryoprotectant. Only NSs containing 5\% (w/v) HP $\beta C D$ did not show signs of aggregation and were easily reconstituted by manual shaking, and the size measurement demonstrated almost identical mean particle size before and after freeze-drying. The cyclic structure of these oligoglucoside compounds ensured a better adsorption onto the NS surface during the sublimation of the aqueous phase. These results are in accordance with those obtained by other authors. ${ }^{39}$

Freeze-drying may generate many stresses that could destabilize colloidal suspension of NSs, especially, the stress of freezing and dehydration. During the freezing step there are dramatic changes in frozen NS formulations. The ice crystallization leads to a phase separation and cryoconcentration of NSs. Such modification can induce aggregation of NSs. Suitable cryoprotectants can prevent such damage. Such excipients can be easily vitrified during freezing and form a protective amorphous matrix around NSs. These sugars are known to vitrify at a specific temperature denoted $\mathrm{T}_{\mathrm{g}}$ (glass transition temperature of maximally cryoconcentrated solutions). In general, freezing must be carried out below $\mathrm{T}_{\mathrm{g}}$, of a frozen sample to ensure the total solidification of the sample. HP $\beta C D$ forms a glassy state at low temperature $\left(T_{g}\right.$, measured by DSC was about $-15^{\circ} \mathrm{C}$ ), a higher value than the other saccharides under study. ${ }^{40}$ These results suggest that lower concentrations of HP $\beta C D$ are needed to maintain particle size.
HP $\beta C D$ forms an amorphous matrix into which the PLGA and PLGA NSs are interdispersed. All particles are well separated throughout the matrix, suggesting the formation of hydrogen bonding between the sugar and the polar groups at the surface of the NSs protecting them during freezing and dehydration. The immobilization of NSs within a glassy matrix of cryoprotectant prevent aggregation of $\mathrm{NSs}^{41}$

Thus, the stabilizing effect of low MW mono- and disaccharides, was not sufficient at the sugar concentration tested, limited by the tonicity of the NS suspensions for ophthalmic administration, which should be around $300 \mathrm{mOsm} / \mathrm{K}$. Results obtained by other authors showed that only $20 \%(\mathrm{w} / \mathrm{v})$ sucrose exerted an acceptable cryoprotectant effect on PLGA NSs. ${ }^{42}$

\section{Influence of $\gamma$-irradiation on the physicochemical characteristics of polymers and NSs}

Freeze-dried NS powders (with and without FB) containing $5 \%(\mathrm{w} / \mathrm{v}) \mathrm{HP} \beta \mathrm{CD}$ as a cryoprotectant agent and the raw PLGA and PLGA-PEG copolymers were $\gamma$-irradiated and the morphometrical properties before and after sterilization were evaluated as can be observed in Table 2. The results obtained demonstrate that $\gamma$-irradiation did not seem to have any effect on particle size, zeta potential, and entrapment efficiency. Reconstitution was achieved by manual shaking.

DSC studies were performed in the raw polymers, and empty and drug-loaded NSs before and after sterilization (Table 3) in order to investigate the physical state of the drug in the NSs, since this aspect could influence the in vitro and in vivo release of the drug from these systems.

PLGA 75:25 raw copolymer exhibited higher $\mathrm{T}_{\mathrm{g}}$ values $\left(59.44^{\circ} \mathrm{C}\right)$ relative to PLGA 50:50 $\left(54.18^{\circ} \mathrm{C}\right)$. The $\mathrm{T}_{\mathrm{g}}$ of PLGA copolymers increase with increasing lactide content 
Table 2 Physicochemical properties and entrapment efficiency of freeze-dried PLGA and PLGA-PEG NSs with and without FB before and after $\gamma$-sterilization with $5 \%(w / v)$ HPßCD

\begin{tabular}{|c|c|c|c|c|c|c|c|c|}
\hline \multirow[t]{2}{*}{ NS composition } & \multicolumn{2}{|c|}{ Size \pm SD (nm) } & \multicolumn{2}{|l|}{$\mathrm{PI} \pm \mathrm{SD}$} & \multicolumn{2}{|c|}{$\zeta$-potential \pm SD $(\mathrm{mV})$} & \multicolumn{2}{|c|}{ EE \pm SD (\%) } \\
\hline & Before* & After & Before* & After & Before* & After & Before* & After \\
\hline PLGA 75:25 & $13.3 \pm 1.3$ & $213.9 \pm 2.5$ & $0.052 \pm 0.034$ & $0.082 \pm 0.020$ & $-23.3 \pm 2.1$ & $-22.9 \pm 1.3$ & - & - \\
\hline PLGA 75:25 + FB & $234.3 \pm 2.1$ & $232.9 \pm 4.0$ & $0.080 \pm 0.007$ & $0.061 \pm 0.013$ & $-23.1 \pm 0.5$ & $-22.5 \pm 0.8$ & $97.1 \pm 1.2$ & $97.0 \pm 1.4$ \\
\hline PLGA 50:50 & $207.9 \pm 0.3$ & $209.4 \pm 5.0$ & $0.036 \pm 0.015$ & $0.081 \pm 0.020$ & $-31.6 \pm 2.1$ & $-32.1 \pm 1.4$ & - & - \\
\hline PLGA 50:50 + FB & $206.1 \pm 2.1$ & $204.9 \pm 4.0$ & $0.083 \pm 0.027$ & $0.101 \pm 0.034$ & $-31.7 \pm 1.1$ & $-31.6 \pm 1.4$ & $91.7 \pm 3.7$ & $92.5 \pm 2.1$ \\
\hline PLGA 50:50-PEG 5\% & $153.2 \pm 1.6$ & $15 \mid .5 \pm 2.8$ & $0.124 \pm 0.008$ & $0.110 \pm 0.015$ & $-20.5 \pm 0.4$ & $-19.9 \pm 1.2$ & - & - \\
\hline PLGA 50:50-PEG 5\% + FB & $150.4 \pm 1.6$ & $150.2 \pm 1.4$ & $0.105 \pm 0.009$ & $0.118 \pm 0.009$ & $-20.4 \pm 0.1$ & $-20.1 \pm 2.0$ & $90.7 \pm 1.8$ & $90.4 \pm 0.8$ \\
\hline PLGA 50:50-PEG I0\% & $126.6 \pm 0.8$ & $124.5 \pm 0.7$ & $0.073 \pm 0.01 \mathrm{I}$ & $0.084 \pm 0.007$ & $-15.2 \pm 0.5$ & $-15.8 \pm 3.2$ & - & - \\
\hline PLGA 50:50-PEG $10 \%+$ FB & $128.8 \pm 1.2$ & $124.9 \pm 0.6$ & $0.084 \pm 0.014$ & $0.089 \pm 0.020$ & $-14.9 \pm 0.1$ & $-13.9 \pm 0.3$ & $83.3 \pm 2.0$ & $83.5 \pm 3.4$ \\
\hline PLGA 50:50-PEG I5\% & $94.5 \pm 0.4$ & $94.5 \pm 0.4$ & $0.115 \pm 0.010$ & $0.130 \pm 0.012$ & $-10.5 \pm 2.3$ & $-10.7 \pm 1.3$ & - & - \\
\hline PLGA 50:50-PEG $15 \%+$ FB & $96.2 \pm 1.1$ & $95.7 \pm 0.3$ & $0.115 \pm 0.014$ & $0.131 \pm 0.004$ & $-10.4 \pm 1.0$ & $-9.9 \pm 1.4$ & $79.6 \pm 0.9$ & $80.3 \pm 1.6$ \\
\hline
\end{tabular}

Notes: Mean \pm SD, $n=3$. *Freeze-dried NSs containing 5\% (w/v) HP $\beta C D(P>0.05$ in size, $\zeta$-potential and EE against nonirradiated samples).

Abbreviations: EE, entrapment efficiency; FB, flurbiprofen; HP $\beta C D$, hydroxypropyl- $\beta$-cyclodextrin; NSs, nanospheres; PI, polydispersity index; PLGA, Poly(D,L-lactide-coglycolide); PLGA-PEG, poly(D,L-lactide-co-glycolide) with poly(ethylene glycol); SD, standard deviation; $\zeta$-potential, zeta potential.

in the copolymer composition and when the MW of the polymer increases, possibly due to a diminution of polymer chain mobility and consequently an increase of $\mathrm{T}_{\mathrm{g}}{ }^{43}$

Results show that the $\mathrm{T}_{\mathrm{g}}$ values of PLGA-PEG copolymers were lower than that obtained for PLGA. $\mathrm{T}_{\mathrm{g}}$ values of polymers depend on their $\mathrm{MW}$ and their composition. Although PLGA-PEG (5\%-15\% of PEG content; see Table 1) copolymers have higher MW than PLGA, they contain PEG chains that cause a slight reduction of the $\mathrm{T}_{\mathrm{g}}$ values of the polymers, due to the plasticizing effect exerted by $\mathrm{PEG}$, which is based on the reduction of the attractive forces among the polymer chains. ${ }^{44}$

Table 3 Values of glass transition temperature for raw polymer, empty NSs, and FB-loaded NSs before and after $\gamma$-irradiation

\begin{tabular}{lll}
\hline & $\mathbf{T}_{\mathbf{g}}\left({ }^{\circ} \mathbf{C}\right)$ & \\
\cline { 2 - 3 } & Nonirradiated & $\gamma$-irradiated \\
\hline PLGA 75:25 raw polymer & 59.44 & $52.84^{*}$ \\
PLGA 75:25 NS & 57.62 & $53.5 I^{*}$ \\
PLGA 75:25 + FB NS & 47.25 & 47.44 \\
PLGA 50:50 raw polymer & 54.18 & $49.78^{*}$ \\
PLGA 50:50 NS & 49.05 & $46.43^{*}$ \\
PLGA 50:50 + FB NS & 44.48 & 45.87 \\
PLGA 50:50-PEG 5\% raw polymer & 49.09 & $34.58^{*}$ \\
PLGA 50:50-PEG 5\% NS & 48.45 & $43.76^{*}$ \\
PLGA 50:50-PEG 5\% + FB NS & 41.41 & 42.54 \\
PLGA 50:50-PEG 10\% raw polymer & 46.09 & $35.99 *$ \\
PLGA 50:50-PEG I0\% NS & 43.33 & $37.95^{*}$ \\
PLGA 50:50-PEG 10\% + FB NS & 42.45 & 40.51 \\
PLGA 50:50-PEG 15\% raw polymer & 43.82 & $33.85^{*}$ \\
PLGA 50:50-PEG 15\% NS & 42.53 & $38.56^{*}$ \\
PLGA 50:50-PEG 15\% + FB NS & 39.55 & 38.78 \\
\hline
\end{tabular}

Note: $* P<0.05$ against nonirradiated samples.

Abbreviations: FB, flurbiprofen; NSs, nanospheres; PLGA, Poly(D,L-lactide-coglycolide); PLGA-PEG, poly(D,L-lactide-co-glycolide) with poly(ethylene glycol); $\mathrm{T}_{\mathrm{g}}$, glass transition temperature.
When empty NSs were tested, the $\mathrm{T}_{\mathrm{g}}$ values obtained were similar to that obtained for the polymers alone, revealing that production process did not affect the copolymer structure. Nevertheless, when the polymer was formulated in NSs containing FB, a lower $\mathrm{T}_{\mathrm{g}}$ was found, probably due to the interaction between FB and the polymer, leading to a plasticization of the polymer. ${ }^{45}$ The melting peak of FB $\left(114.9^{\circ} \mathrm{C}\right)$ was not detected in all NSs analyzed, suggesting the absence of free crystalline FB. It can thus be concluded that FB formulated in PLGA and PLGA-PEG NSs was in an amorphous or disordered crystalline phase of a molecular dispersion or a solid solution state in the polymer matrix.

As shown in Table 3, $\gamma$-irradiation of raw polymers and empty NSs caused a small reduction in the $\mathrm{T}_{\mathrm{g}}$ values $(P<0.05)$. This effect may be due to the chain scission of the polymers during the sterilization process, which led to a decrement of MW of polymers and consequently an increase of free volume and chain mobility ${ }^{46}$ Nevertheless, $\mathrm{T}_{\mathrm{g}}$ values of sterilized and nonsterilized FB-loaded NSs were similar $(P>0.05)$. FB incorporated into the polymeric matrix seems to play an important role on NSs behavior after irradiation, improving PLGA and PLGA-PEG NSs stability.

The evaluation of the possible degradation of FB by irradiation was performed by HPLC. The chromatograms extracted from the irradiated samples were similar to those of the nonirradiated samples and did not reveal any evidence of the drug radiolysis at the irradiation dose tested.

\section{In vitro release of NSs}

Saturation concentration of FB in the release medium (isotonic PBS $0.1 \mathrm{M}, \mathrm{pH} 7.4$ ) at $32^{\circ} \mathrm{C}$ is $4.86 \pm 0.3 \mathrm{mg} / \mathrm{mL}$, 


\section{A}

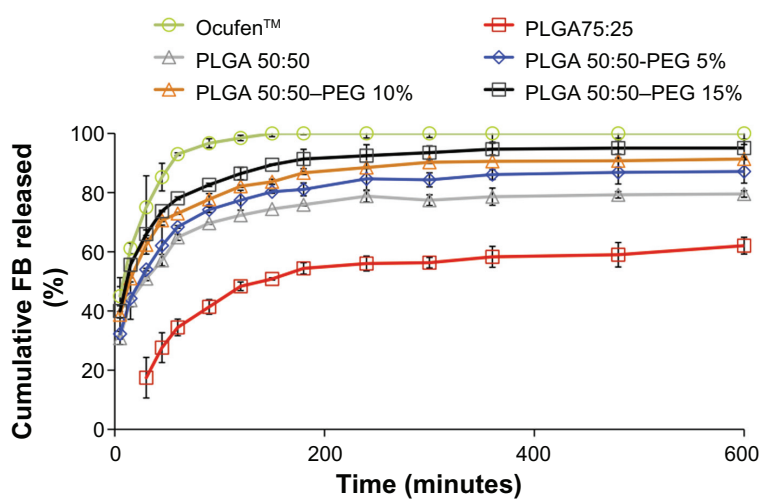

B

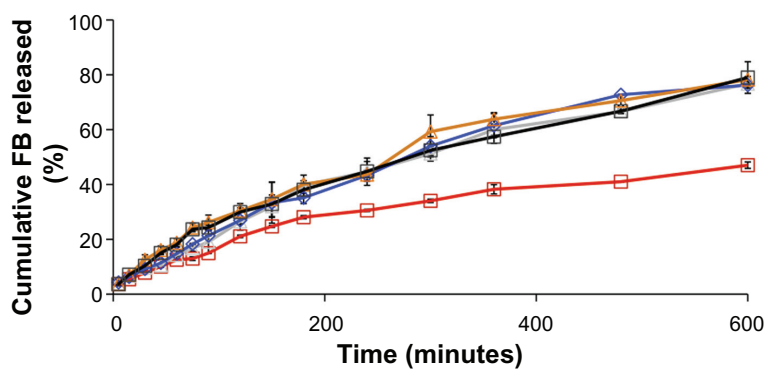

C

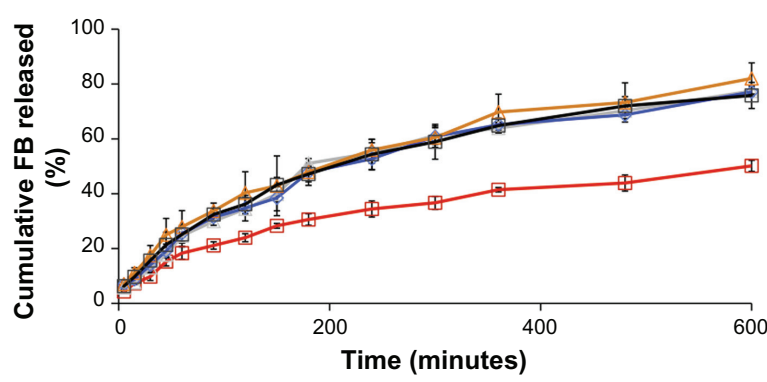

Figure 2 In vitro release profiles of FB from: (A) PLGA and PLGA-PEG NSs, (B) PLGA and PLGA-NSs in the presence of $5 \%(w / v)$ HPßCD, (C) PLGA and PLGA-NSs in the presence of $5 \%(w / v)$ HPBCD after sterilization.

Note: Error bars represent \pm SD.

Abbreviations: FB, flurbiprofen; HP $\beta C D$, hydroxypropyl- $\beta$-cyclodextrin; NS, nanospheres; PLGA, Poly(D,L-lactide-co-glycolide); PLGA-PEG, poly(D,L-lactideco-glycolide) with poly(ethylene glycol); SD, standard deviation. which demonstrates that the "sink conditions" were maintained during the whole experiment.

The cumulative in vitro release profiles of PLGA and PLGA-PEG NSs are given in Figure 2. For all formulations, release occurred in two phases: an initial burst release, attributed to the fraction of drug that was adsorbed on the surface of the NSs, followed by a more gradual release. Release was slower for PLGA 75:25 than PLGA 50:50 (34.4\% $\pm 2.8 \%$ and $64.9 \% \pm 1.1 \%$, respectively) in the first hour of study, probably due to the more hydrophobic nature due to increase in lactide content of the polymer and high drug loading of the FB due to the higher affinity of the drug with the polymer, which results in a slower diffusion of the FB out of the polymer matrix and showing a more sustained release.

As can be observed PLGA-PEG NSs resulted in significantly faster release of the drug from the NSs compared with that from PLGA samples. This might be due to porous morphology of the PLGA-PEG NSs and the drug being partially entrapped within the PEG chains, which facilitates FB release. ${ }^{47}$

The effect of incorporating HP $\beta B C$ in PLGA and PLGAPEG NSs was evaluated. The addition of HPßBC significantly decreased burst release of FB probably due to the interaction of HP $\beta B C$ onto the surface of the NSs, which creates a diffusional resistance to the release of the drug creating an additional barrier to the release of the drug. ${ }^{37}$

In this study, the Korsmeyer-Peppas model was used to analyze the release data. The diffusional exponent (n), determination coefficient $\left(\mathrm{r}^{2}\right)$, and the release rate constant (k) obtained are summarized in Table 4. Good determination coefficients $\left(r^{2}\right)$ between 0.9404 and 0.9989 were obtained. It was shown that the exponent $\mathrm{n}$ values for the FB release from NSs without $\mathrm{HP} \beta C D$ were less than 0.43 , suggesting Fickian release behavior. ${ }^{48}$

Table 4 Release kinetics obtained after fitting FB release data from NSs into Korsmeyer-Peppas model

\begin{tabular}{|c|c|c|c|c|}
\hline \multirow[t]{2}{*}{ NS composition } & \multicolumn{3}{|c|}{ Korsmeyer-Peppas model } & \multirow[t]{2}{*}{ Transport mechanism } \\
\hline & $\overline{r^{2}}$ & $k \pm S D\left(h^{-n}\right)$ & $\mathrm{n} \pm \mathrm{SD}$ & \\
\hline PLGA 75:25 & 0.9637 & $1.64 \pm 0.38$ & $0.28 \pm 0.04$ & Fickian diffusion \\
\hline PLGA 50:50 & 0.9451 & $4.59 \pm 0.45$ & $0.16 \pm 0.02$ & Fickian diffusion \\
\hline PLGA 50:50-PEG 5\% & 0.9404 & $5.02 \pm 0.51$ & $0.16 \pm 0.02$ & Fickian diffusion \\
\hline PLGA 50:50-PEG I0\% & 0.9563 & $5.65 \pm 0.44$ & $0.15 \pm 0.01$ & Fickian diffusion \\
\hline PLGA 50:50-PEG I5\% & 0.9511 & $5.49 \pm 0.49$ & $0.16 \pm 0.02$ & Fickian diffusion \\
\hline PLGA 75:25/HP $\beta C D$ & 0.9922 & $0.21 \pm 0.03$ & $0.56 \pm 0.03$ & Non-Fickian or anomalous diffusion \\
\hline PLGA 50:50/HP $\beta C D$ & 0.9945 & $0.15 \pm 0.02$ & $0.68 \pm 0.03$ & Non-Fickian or anomalous diffusion \\
\hline PLGA 50:50-PEG 5\%/HP $\beta C D$ & 0.9950 & $0.15 \pm 0.02$ & $0.68 \pm 0.03$ & Non-Fickian or anomalous diffusion \\
\hline PLGA 50:50-PEG I0\%/HPßCD & 0.9949 & $0.26 \pm 0.03$ & $0.60 \pm 0.02$ & Non-Fickian or anomalous diffusion \\
\hline PLGA 50:50-PEG I5\%/HPßCD & 0.9989 & $0.23 \pm 0.01$ & $0.62 \pm 0.01$ & Non-Fickian or anomalous diffusion \\
\hline
\end{tabular}

Note: Mean \pm SD, $n=3$.

Abbreviations: FB, flurbiprofen; HP $\beta C D$, hydroxypropyl- $\beta$-cyclodextrin; $k$, release rate constant; $n$, diffusional exponent; NSs, nanospheres; PLGA, Poly(D,L-lactide-coglycolide); PLGA-PEG, poly(D,L-lactide-co-glycolide) with poly(ethylene glycol); SD, standard deviation. 
However, when HP $\beta C D$ was incorporated into the formulations, results obtained show anomalous (non-Fickian) release. ${ }^{49}$ This may be due to the reservoir effect of HP $\beta C D$, which causes a sustained release of FB.

The release profile of $\gamma$-irradiated NSs showed a similar biphasic pattern in both irradiated and nonirradiated samples. Both profiles were compared using the similarity factor $\left(\mathrm{f}_{2}\right)$, which allows determination of dissolution profiles (it was estimated that a value for the similarity factor between 50 and 100 indicated similarity between curves). The $f_{2}$ values obtained for PLGA 75:25, PLGA 50:50, PLGA 50:50-PEG 5\%, PLGA 50:50-PEG 10\%, and PLGA 50:50-PEG 15\% were $73.87,56.12,56.95,57.11$, and 58.05, respectively, indicating that the curves were similar and overlapping. These results demonstrated that $\gamma$-irradiation did not influence drug release kinetics.

\section{Ex vivo study CLSM}

NSs prepared using Rd showed high entrapment efficiency (around 98\% for all formulations) and the in vitro release experiment indicated that only negligible amounts of $\mathrm{Rd}$ were released from NSs in a 2-hour period (10\%). Consequently, $\mathrm{Rd}$ is an adequate marker that remains associated to the system for extended periods of time.

The images obtained (Figure 3 ) by CLSM of the rabbit corneal epithelium (ex vivo study) exposed to Rd-NSs (PLGA and PLGA-PEG NSs with and without HP $\beta C D$ ), showed that the fluorescent spots are uniformly distributed inside the cells, rather than in the intercellular spaces, thus suggesting that the NSs penetrate the corneal epithelium through a transcellular pathway. Control images in the absence of NSs did not show fluorescent in the cells (data not shown). These results are in accordance with those reported previously for poly(alkylcyanoacrylate) ${ }^{50}$ and poly- $\varepsilon$-caprolactone nanoparticles. ${ }^{51}$ The mechanism of cellular internalization is thought to occur predominantly by adsorptive endocytosis indicated by nonspecific interactions between nanoparticles and cell membranes in the first layers of the corneal epithelium. ${ }^{52}$

\section{Corneal permeation studies}

In order to evaluate the capacity of the developed systems to enhance the corneal penetration of $\mathrm{FB}$, an ex vivo study was performed (Figure 4). The apparent corneal permeability coefficient $\left(\mathrm{P}_{\text {app }}\right)$ and hydration level of each formulation is shown in Table 5. Results for PLGA 75:25, free FB in isotonic PBS, and commercial eyedrops $\left(\right.$ Ocufen $\left.^{\circledR}\right)$ were obtained from
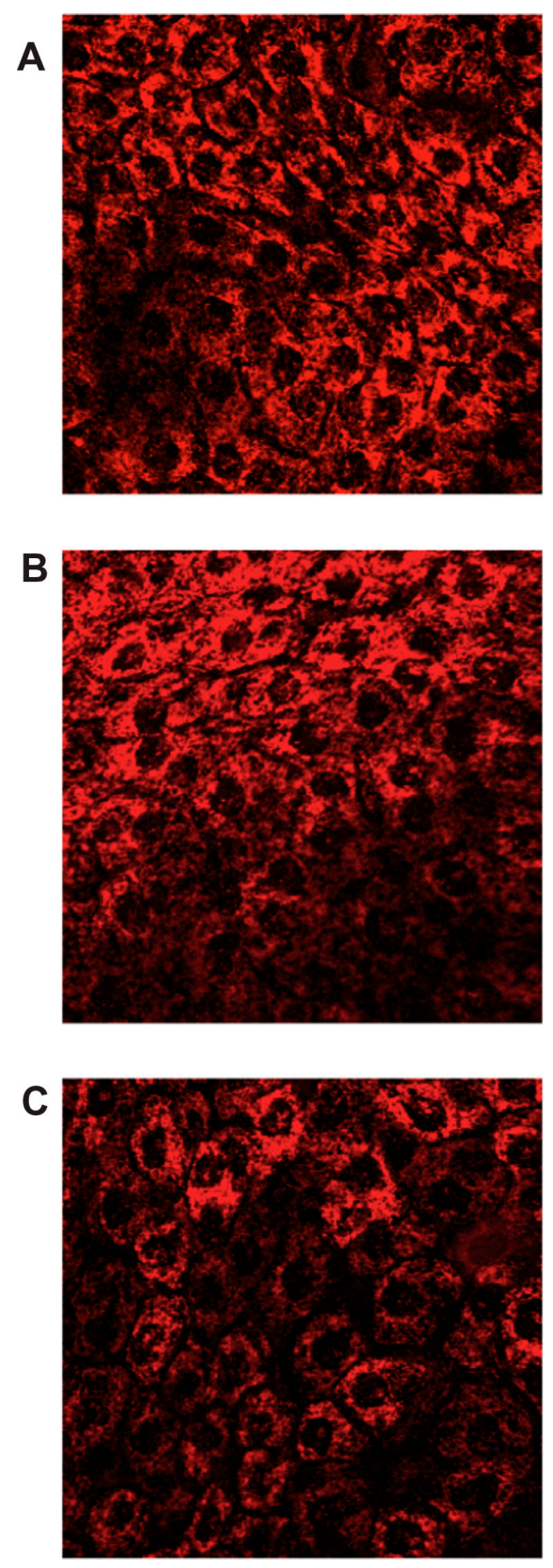

Figure 3 Confocal images of a cross-section of the corneal epithelium at depth of $10 \mu \mathrm{m}$ after 2 hours of incubation with: (A) Rd-PLGA NS; (B) Rd-PEG-PLGA NSs; (C) Rd-NSs containing 5\% (w/v) HP $\beta C D$.

Abbreviations: HP $\beta C D$, hydroxypropyl- $\beta$-cyclodextrin; NS, nanospheres; PLGA, Poly(D,L-lactide-co-glycolide); PLGA-PEG, poly(D,L-lactide-co-glycolide) with poly(ethylene glycol); Rd, rhodamine-6G.

previous studies. ${ }^{5}$ PLGA and PLGA-PEG NSs containing $1.5 \%$ $\mathrm{FB}\left(\mathrm{w} / \mathrm{v}\right.$ ) showed $\mathrm{P}_{\text {app }}$ values (Table 5) higher than those obtained previously for free $\mathrm{FB}$ in isotonic $\mathrm{PBS}$ and commercial eyedrops $\left(\right.$ Ocufen $\left.^{\circledR}\right)$ with $0.3 \%(\mathrm{w} / \mathrm{v}) \mathrm{FB}\left(2.48 \pm 1.06 \times 10^{-6} \mathrm{~cm} \cdot \mathrm{s}^{-1}\right.$ and $11.28 \pm 5.26 \times 10^{-6} \mathrm{~cm} \cdot \mathrm{s}^{-1}$, respectively).

The $\mathrm{P}_{\text {app }}$ values were found to be similar for the NSs prepared with different ratios of lactic acid/glycolic acid $(P>0.05)$. Both PLGA 75:25 and PLGA 50:50 are useful 


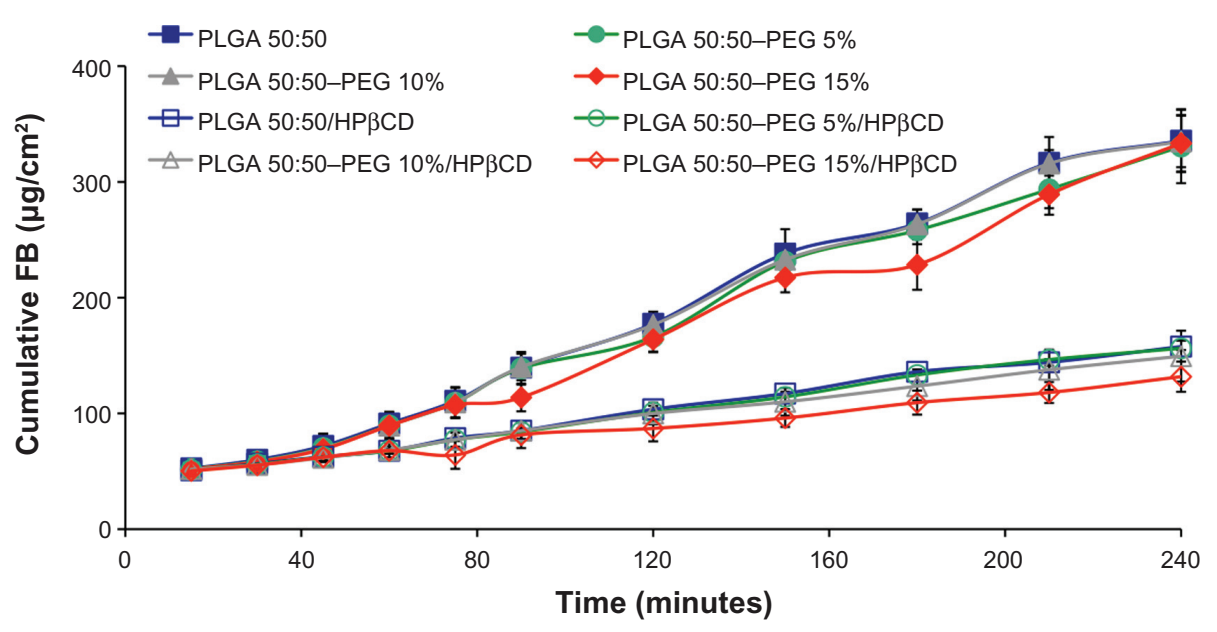

Figure 4 Permeation of FB through isolated rabbit cornea from PLGA and PLGA-PEG NSs with and without HPBCD.

Note: Error bars represent \pm SD.

Abbreviations: FB, flurbiprofen; HP $\beta C D$, hydroxypropyl- $\beta$-cyclodextrin; NS, nanospheres; PLGA, Poly(D,L-lactide-co-glycolide); PLGA-PEG, poly(D,L-lactide-co-glycolide) with poly(ethylene glycol); SD, standard deviation.

in terms of increasing the penetration of FB and no influence of the composition of the copolymer was observed.

The effect of PEG on the transcorneal permeation of FB was evaluated as shown in Figure 4. PEG-PLGA NSs showed no differences compare to PLGA NSs $(P>0.05)$. The presence of the PEG steric barrier is mainly due to prevent the adsorption of enzymes and proteins, such as lysozyme, an enzyme that is highly concentrated in the mucus ocular layer and mucus components, thereby enhancing the stability in contact with mucosal fluids and, hence, improving the interaction of these colloidal systems with the ocular mucosa. ${ }^{53}$ However, the ex vivo study was performed in the absence of enzymes and mucin and, consequently, the expected positive effect of PEG coating was not evident. ${ }^{54}$ Results obtained in vivo by other authors demonstrated the increase in ocular bioavailability by PEG coating of these colloidal systems. ${ }^{8}$

Table 5 Corneal hydration levels and permeation coefficients of different NS formulations containing FB

\begin{tabular}{|c|c|c|}
\hline NS composition & HL (\%) & $P_{\text {app }}\left(\times 10^{-6} \mathrm{~cm} \cdot \mathrm{s}^{-1}\right)$ \\
\hline PLGA 50:50 & $77.95 \pm 1.06$ & $17.72 \pm 2.54$ \\
\hline PLGA 50:50-PEG 5\% & $79.01 \pm 3.25$ & $17.15 \pm 5.12$ \\
\hline PLGA 50:50-PEG I0\% & $78.43 \pm 1.99$ & $17.60 \pm 4.65$ \\
\hline PLGA 50:50-PEG I5\% & $78.64 \pm 2.67$ & $17.07 \pm 3.14$ \\
\hline PLGA 75:25/HPßCD & $79.15 \pm 2.15$ & $8.73 \pm 3.97$ \\
\hline PLGA 50:50/HPßCD & $78.16 \pm 2.65$ & $8.80 \pm 3.62$ \\
\hline PLGA 50:50-PEG 5\%/HP $\beta C D$ & $79.46 \pm 1.68$ & $9.67 \pm 1.95$ \\
\hline PLGA 50:50-PEG I0\%/HPßCD & $77.56 \pm 1.04$ & $9.19 \pm 1.39$ \\
\hline PLGA 50:50-PEG I5\%/HPßCD & $78.26 \pm 0.95$ & $8.45 \pm 3.97$ \\
\hline
\end{tabular}

Note: Mean $\pm S D, n=6$.

Abbreviations: $\mathrm{FB}$, flurbiprofen; $\mathrm{HL}$, corneal hydration level; $\mathrm{HP} \beta C D$, hydroxypropyl- $\beta$-cyclodextrin; NS, nanospheres; $P_{\text {app }}$, permeation coefficient; PLGA, Poly(D,L-lactide-co-glycolide); PLGA-PEG, poly(D,L-lactide-co-glycolide) with poly(ethylene glycol); SD, standard deviation.
Results showed that there was a significant decrease in $\mathrm{P}_{\text {app }}$ when HP $\beta C D$ was added to the formulations. This might be related to the slower diffusion of drug from NSs as can be observed from the results obtained in the in vitro release study giving a more sustained release of the drug from the colloidal systems, due to the complex formation between nonentrapped FB and the HP $\beta C D .{ }^{55,56}$ The results suggest two mechanisms of penetration of the corneal membrane: NSs containing the entrapped drug penetrate the first layers of the corneal epithelium as demonstrated in the images obtained by CLSM. However, free FB not entrapped into the polymeric network forms a complex with HP $\beta C D$. In this latter case, HP $\beta C D$ acts as a true carrier by keeping FB and delivering the drug to the surface of the ocular barrier where FB, which is a small (MW = 244 D) and highly hydrophobic drug, penetrates through the cornea mainly by passive transcellular diffusion. ${ }^{57}$ The cornea has low affinity for the hydrophilic HP $\beta C D$ molecules and therefore they remain in the aqueous exterior membrane. ${ }^{58}$ Corneal hydration is frequently used as a parameter to evaluate damage to this tissue. The normal cornea has a hydration level of $76 \%-80 \%{ }^{57} \mathrm{~A}$ hydration level that is $3 \%-7 \%$ units above the normal value denotes damage to the epithelium or endothelium. ${ }^{59}$ As shown in Table 5, corneal hydration after the studies was not increased by more than $3 \%$, indicating that the formulations did not cause any damage to the epithelium or endothelium during the studies.

\section{Ocular tolerance} In vitro Eytex ${ }^{\mathrm{TM}}$ test

FB-loaded NSs showed IDE values ranging between 7.8 and 9.7 and was classified into the rank of "minimal" irritancy. 


\section{In vivo Draize test}

Since a single in vitro test could not mimic the entire situation in vivo, tolerance studies on male albino rabbits were performed. Topical application of formulations to rabbit eyes showed no sign of toxicity or irritation to the external ocular tissues (MTS $=0$ in all cases) after the Draize test, which are in accordance with the results obtained by the Eytex ${ }^{\mathrm{TM}}$ test.

\section{Long term stability of NSs}

To ensure NSs stability of the $\gamma$-irradiated and freeze-dried NS powders, a storage stability study was performed.

A

$\square$ Before irradiation

$\square \mathrm{t}=0$

6 months $\left(4^{\circ} \mathrm{C}\right)$

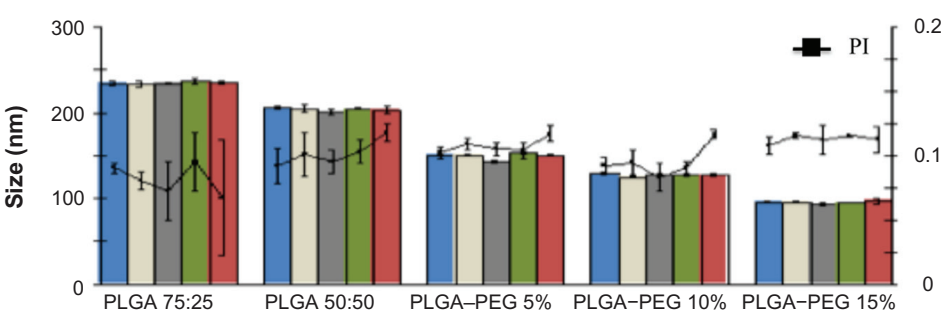

B

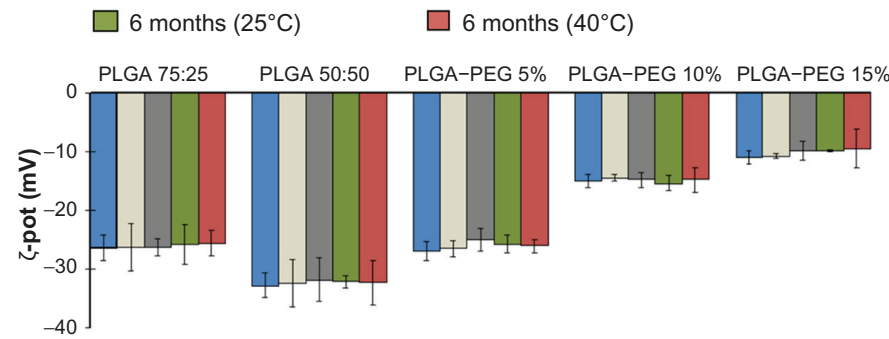

C

$\square 1$ month $\left(4^{\circ} \mathrm{C}\right) \quad \square 3$ months $\left(4^{\circ} \mathrm{C}\right) \quad \square 1$ month $\left(25^{\circ} \mathrm{C}\right)$

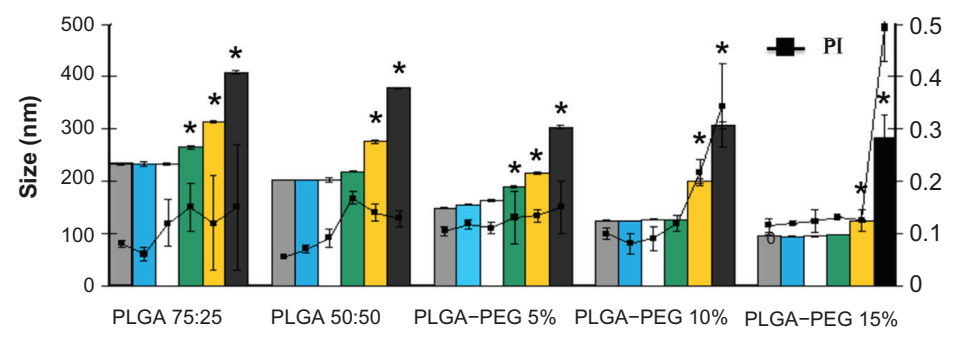

D

3 months $\left(25^{\circ} \mathrm{C}\right)$

1 month $\left(40^{\circ} \mathrm{C}\right)$

3 months $\left(40^{\circ} \mathrm{C}\right)$

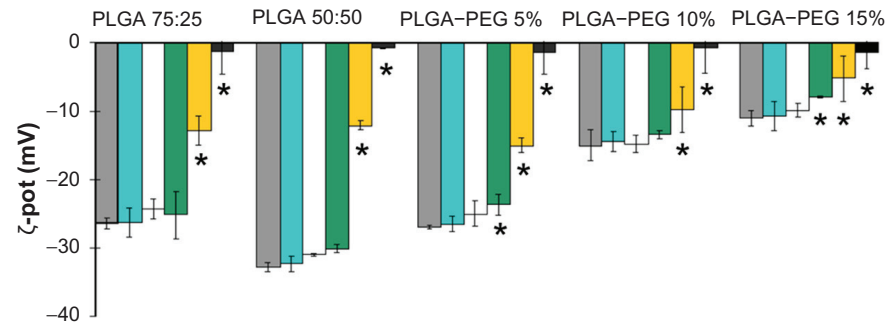

Figure 5 (A and B) Particle size, PI, and $\zeta$-pot of the sterilized NS powders stored at $4{ }^{\circ} \mathrm{C}, 25^{\circ} \mathrm{C}$, and $40^{\circ} \mathrm{C}$ after 6 months of storage; $(\mathbf{C}$ and $\mathbf{D})$ Particle size, PI, and $\zeta$-pot of the rehydrated samples after I and 3 months of storage at $4{ }^{\circ} \mathrm{C}, 25^{\circ} \mathrm{C}$, and $40^{\circ} \mathrm{C}$.

Notes: Error bars represent $\pm \mathrm{SD} ; * \mathrm{P}<0.05$.

Abbreviations: HPßCD, hydroxypropyl- $\beta$-cyclodextrin; NS, nanospheres; PI, polydispersity index; PLGA, Poly(D,L-lactide-co-glycolide); PLGA-PEG, poly(D,L-lactide-coglycolide) with poly(ethylene glycol); SD, standard deviation; $\zeta$-pot, zeta potential. 


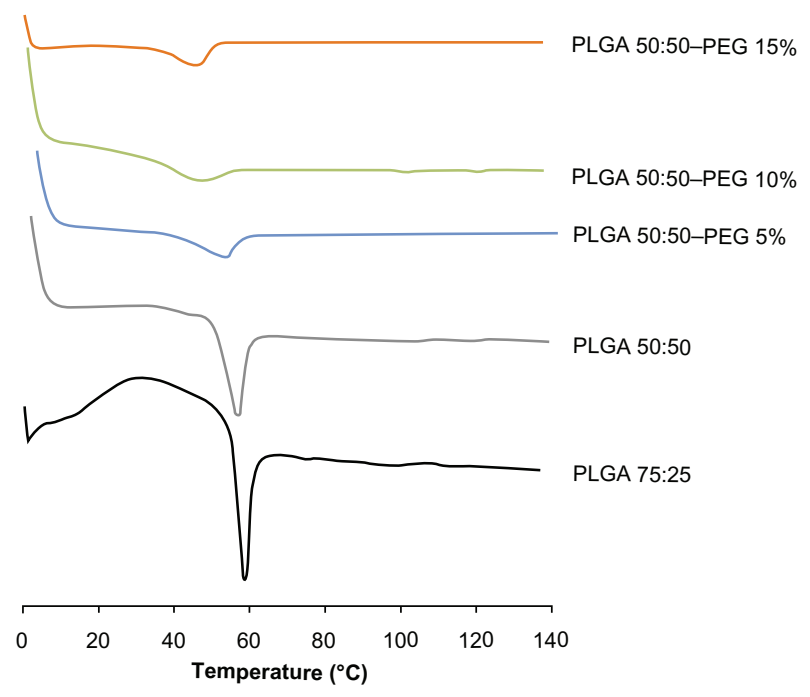

Figure 6 DSC thermograms of $\gamma$-irradiated PLGA and PLGA-PEG NSs containing $5 \%(w / v) H P \beta C D$ stored 6 months at $4^{\circ} \mathrm{C}$ and after rehydrated and stored for 3 months in the aqueous form at the same temperature.

Abbreviations: DSC, differential scanning calorimetry; HP $\beta C D$, hydroxypropyl$\beta$-cyclodextrin; NS, nanospheres; PLGA, Poly(D,L-lactide-co-glycolide); PLGA-PEG, poly(D,L-lactide-co-glycolide) with poly(ethylene glycol).

NSs were stored at $4{ }^{\circ} \mathrm{C}, 25^{\circ} \mathrm{C}$, and $40^{\circ} \mathrm{C}$. After 6 months of storage, three samples of each lyophilized NS formulation were reconstituted and the physicochemical properties and the entrapment efficiency were determined. All formulations maintained their initial properties $(P>0.05)$ and could be easily resuspended by manual shacking after 6 months of storage at $4^{\circ} \mathrm{C}, 25^{\circ} \mathrm{C}$, and $40^{\circ} \mathrm{C}$ (Figure 5).

The stability of the rehydrated NSs powders was determined at $4^{\circ} \mathrm{C}, 25^{\circ} \mathrm{C}$, and $40^{\circ} \mathrm{C}$ for 3 months of storage. Only formulations stored at $4^{\circ} \mathrm{C}$ remained unchanged in the aqueous form $(P>0.05)$. A slight increase in particle size and a decrease in zeta potential values were found for NSs stored at $25^{\circ} \mathrm{C}$, which remained still suitable for ophthalmic administration at this temperature. However, a significant aggregation of the NSs was found at $40^{\circ} \mathrm{C}(P<0.05)$. At this temperature, polymers were in the rubbery state. In this state, a polymer is at a higher free energy level than in its glassy state and thus is more reactive and the degradation is faster. A progressive decrease in $\mathrm{pH}$ values and an increase of tonicity appeared in the formulations stored at $40^{\circ} \mathrm{C}$, which could be related to the oligomers release by polymer hydrolysis. ${ }^{60}$

Thermal properties were determined during storage conditions. As shown in Figure 6, no endothermic peak corresponding to the drug melting was detected in dried formulations stored for 6 months at $4{ }^{\circ} \mathrm{C}$ and rehydrated and stored for 3 months at the same temperature.

\section{Conclusion}

In this work, we report the possibility of combining the advantages of polymeric NSs in terms of increasing FB permeability through corneal epithelium with the solubilizing properties of $\mathrm{HP} \beta C D$ in order to be an effective system for increasing the bioavailability of FB. Freeze-drying and sterilization of these formulations by a suitable method is often problematic. This work demonstrates that $\mathrm{HP} \beta \mathrm{BC}$ is a suitable cryoprotectant in order to maintain the properties of the NSs developed and $\gamma$-irradiation allows sterile formulations to be obtained for ophthalmic administration preventing contamination of these formulations.

\section{Acknowledgment}

This work was supported by the Spanish Ministry of Science and Technology (MCyT projects MAT2011-26994 and MAT2010-19877).

\section{Disclosure}

The authors report no conflict of interest in this work.

\section{References}

1. Lee VHL, Robinson JR. Mechanistic and quantitative evaluation of precorneal pilocarpine disposition in albino rabbits. J Pharm Sci. 1979;68:678-684.

2. Lang JC. Ocular drug delivery conventional ocular formulations. $A d v$ Drug Delivery Rev. 1995;16:39-43.

3. Araújo J, Gonzalez E, Egea MA, Garcia ML, Souto EB. Nanomedicines for ocular NSAIDS: safety on drug delivery. Nanomedicine. 2009;5:394-401.

4. Vega E, Egea MA, Valls O, Espina M, García ML. Flurbiprofen loaded biodegradable nanoparticles for ophthalmic administration. J Pharm Sci. 2006;95:2393-2405.

5. Vega E, Gamisans F, García ML, Chauvet A, Lacoulonche F, Egea MA. PLGA nanospheres for the ocular delivery of flurbiprofen: drug release and interactions. J Pharm Sci. 2008;97:5306-5317.

6. Dillen K, Weyenberg W, Vandervoort J, Ludwig A. The influence of the use of viscosifying agents as dispersion media on the drug release properties from PLGA nanoparticles. Eur J Pharm Biopharm. 2004;58:539-549.

7. Anderson JM, Shive MS. Biodegradation and biocompatibility of PLA and PLGA microspheres. Adv Drug Delivery Rev. 1997;28:5-24.

8. Giannavola C, Bucolo C, Maltese A, et al. Influence of preparation conditions on acyclovir-loaded poly-d,l-Lactic acid nanospheres and effect of PEG coating on ocular drug bioavailability. Pharm Res. 2003;20:584-590.

9. Abdelwahed W, Degobert G, Stainmesse S, Fessi H. Freeze-drying of nanoparticles: formulation, process and storage considerations. $A d v$ Drug Delivery Rev. 2006;58:1688-1713.

10. Athanasiou KA, Niederauer GG, Agrawal CM. Sterilization, toxicity, biocompatibility and clinical applications of polylactic acid/polyglycolic acid copolymers. Biomaterials. 1996;17:93-102.

11. Yoshioka S, Aso Y, Otsuka T, Kojima S. The effect of $\gamma$-irradiation on drug release from poly(lactide) microspheres. Radiat Phys Chem. 1995;46:281-285.

12. European Guideline 3AQ4a. The Use of Ionizing Radiation in the Manufacture of Medicinal Products. London, UK: Official Publications in the European Communities; 1992. 
13. Hausberger AG, Kenley RA, DeLuca PP. Gamma irradiation effects on molecular weight and in vitro degradation of poly(D,L-lactide-coglycolide) microparticles. Pharm Res. 1995;12:233-242.

14. Sintzel MB, Merkli A, Tabatabay C, Gurny R. Influence of irradiation sterilization on polymers used as drug carriers - A review. Drug Dev Ind Pharm. 1997;23:857-879.

15. Montanari L, Costantini M, Ciranni-Signoretti E, et al. Gamma irradiation effects on poly(D,L-lactide-co-glycolide) microspheres. J Control Release. 1998;56:219-229.

16. De Rosa G, Larobina D, Immacolata La Rotonda M, et al. How cyclodextrin incorporation affects the properties of protein-loaded PLGAbased microspheres: the case of insulin/hydroxypropyl- $\beta$-cyclodextrin system. J Control Release. 2005;102:71-83.

17. Bibby DC, Davies IG, Tucker IG. Mechanism by which cyclodextrins modify drug release from polymeric drug delivery systems. J Control Release. 2000;62:263-268.

18. Van Sorge AA, Wijnen PH, Van Delft JL, Coré-Bodelier VM, van Haeringen NJ. Flurbiprofen, S(+), eyedrops: formulation, enantiomeric assay, shelflife and pharmacology. Pharm World Sci. 1999;21:91-95.

19. Fessi H, Puisieux F, Devissaguet JP, Ammoury N, Benita S. Nanocapsule formation by interfacial polymer deposition following solvent displacement. Int J Pharm. 1989;55:R1-R4.

20. Albert KS, Raabe A, Garry M, Antal EJ, Gillespie WR. Determination of ibuprofen in capillary and venous plasma by high-performance liquid chromatography with ultraviolet detection. J Pharm Sci. 1984;73:1487-1489.

21. Thomas ML, Szeto VR, Gan CM, Polse KA. Sequential staining: The effects of sodium fluorescein, osmolality, and $\mathrm{pH}$ on human corneal epithelium. Optom Vision Sci. 1997;74:207-210.

22. Baeyens V, Varesio E, Veuthey JL, Gurny R. Determination of dexamethasone in tears by capillary electrophoresis. J Chrom Biomed. 1997;692:222-226.

23. Levy MY, Benita S. Drug release from submicronized o/w emulsion: A new in vitro kinetic evaluation model. Int J Pharm. 1990;66: 29-37.

24. Korsmeyer RW, Gurny R, Doelker E, Buri P, Peppas NA. Mechanisms of solute release from porous hydrophilic polymers. Int J Pharm. 1983;5:25-35.

25. US Department of Health and Human Services. Guidedance for Industry: Dissolution testing of immediate release solid dosage forms. Rockville, MD: US Department of Health and Human Services; 1997.

26. Gordon VC. Utilization of biomacromolecular in vitro assay systems in the prediction of in vivo toxic responses. Lens Eye Toxic Res. 1992;9:211-227.

27. Soto RJ, Gordon VC. An in vitro method for estimating ocular irritation. Toxicol Vitro. 1990;4:332-335.

28. Fielder RJ. Acceptance of in vitro studies by regulatory authorities. Toxicol Vitro. 1994;8:911-916.

29. Duprat P, Delsaut L, Gradiski D. Irritant power of the principle chlorinated aliphatic solvents on the skin and ocular mucosa of the rabbit. Eur J Toxicol Environ Hyg. 1976;9:171-177.

30. Kay JH, Calandra JC. Interpretation of eye irritation tests. J Soc Cosmet Chem. 1962;13:281-289.

31. Passantino A. Application of the 3 Rs principles for animals used for experiments at the beginning of the 21st century. Ann Rev Biomed Sci. 2008; 10:27-32.

32. Schoenwald RD, Stewart P. Effect of particle size on ophthalmic bioavailability of dexamethasone suspensions in rabbits. J Pharm Sci. 1979;69:391-394.

33. Dalwadi G, Sunderland B. An ion pairing approach to increase the loading of hydrophilic and lipophilic drugs into PEGylated PLGA nanoparticles. Eur J Pharm Biopharm. 2009;71:231-242.

34. Celikkaya E, Denkbas EB, Piskin E. Rifampicin carrying poly(D,Llactide)/poly(ethylene glycol) microspheres: loading and drug release. Artif Organs. 1996;20:743-751.
35. Govender T, Stolnik S, Garnett MC, Illum L, Davis SS. PLGA nanoparticles prepared by nanoprecipitation: Drug loading and release studies of a water soluble drug. J Control Release. 1999;57:171-185.

36. Muller RH, Wallis KH. Surface modification of iv injectable biodegradable nanoparticles with poloxamer polymers and poloxamine 908. Int J Pharm. 1993;89:25-31.

37. Silveira AM, Ponchel G, Puisieux F, Duchêne D. Combined poly(isobutylcyanoacrylate) and cyclodextrins nanoparticles for enhancing the encapsulation of lipophilic drugs. Pharm Research. 1998;15:1051-1055.

38. Quintanar-Guerrero D, Ganem-Quintanar A, Allémann A, Fessi H, Doelker E. Influence of the stabilizer coating on the purification and freeze-druing of poly (D,L-lactic acid) nanoparticles prepared by an emulsion-diffusion technique. J Microencapsul. 1998;15:107-119.

39. Musumeci T, Vicari L, Ventura C, Gulisano M, Pignatello R, Puglisi G. Lyoprotected nanospheres formulations for paclitaxel controlled delivery. J Nanosci Nanotechnol. 2006;6:3118-3125.

40. Abdelwahed W, Degobert G, Fessi H. A pilot study of freeze drying of poly(epsilon-caprolactone) nanocapsules stabilized by poly(vinyl alcohol): Formulation and process optimization. Int J Pharm. 2005;309:178-188.

41. Crowe JH, Crowe LM. Preservation of liposomes by freeze-drying. In: Gregoriadis G, editors. Liposome Technology. Boca Raton: CRC Press; 1992:229-52.

42. Saez A, Guzmán M, Molceperes J, Aberturas MR. Freeze-drying of polycaprolactone and poly(D,L-lactic-glycolic) nanoparticles induce minor particle size changes affecting the oral pharmacokinetics of loaded drugs. Eur J Pharm Biopharm. 2000;50:379-387.

43. Wang N, Wu S, Shen L, Feng MF. Synthesis, characterization, biodegradation, and drug delivery application of biodegradable lactic/glycolic acid polymers: I. Synthesis and characterization. J Biomaterials Sci. 2000;11:301-318.

44. Kulinski Z, Piorkowska E, Gadzinowska K, Stasiak M. Plasticization of poly(1-lactide) with poly(propylene glycol). Biomacromolecules. 2006; 7:2128-2135.

45. Dubernet C. Thermoanalysis of microspheres. Termochimica Acta. 1995;248:259-269.

46. Bittner B, Mäder K, Kroll C, Borchert HH, Kissel T. Tetracycline-HClloaded poly(D,L-lactide-co-glycolide) microspheres prepared by a spray drying technique: influence of gamma-irradiation on radical formation and polymer degradation. J Control Release. 1999;59:23-32.

47. Lewis DH. Controlled release of bioactive agents from lactide glycolide polymers. In: Chasin M, Langer R, editors. Biodegradable Polymers as Drug-Delivery Systems. New York, NY: Marcel Dekker; 1990:1-40.

48. Peppas NA. Analysis of fickian and non-fickian drug release from polymers. Pharms Acta Helv. 1985;60:110.

49. Mundargi RC, Shelke NB, Aminabhavi TM. Formulation and in vitro evaluation of novel starch-based tableted microspheres for controlled release of ampicilin. Carbohydr Polym. 2008;71:42-53.

50. Calvo P, Thomas C, Alonso MJ, Vila Jato JL, Robinson J. Study of the mechanism of interaction of poly- $\varepsilon$-caprolactone nanocapsules with the cornea by confocal laser scanning microscopy. Int J Pharm. 1994;103:283-291.

51. Wood RW, Li VH, Kreuter R, Robinson JR. Ocular disposition of polyhexyl-2-cyano-[3- $\left.{ }^{14} \mathrm{C}\right]$ acrylate nanoparticles in the albino rabbit. Int J Pharm. 1985;23:175.

52. Qaddoumi MG, Ueda H, Yang J, Davda J, Labhasetwar V, Lee VH. The characteristics and mechanisms of uptake of PLGA nanoparticles in rabbit conjunctival epithelial cell layers. Pharm Res. 2004;21:641-648.

53. Fresta M, Fontana G, Bucolo C, Cavallaro G, Giammona G, Puglisi G. Ocular tolerability and in vivo bioavailability of poly(ethylene glycol) (PEG)-coated polyethyl-2-cyanoacrylate nanosphere encapsulated acyclovir. J Pharm Sci. 2001;90:288-297.

54. De Campos AM, Sanchez A, Gref R, Calvo P, Alonso MJ. The effect of a PEG versus Chitosan coating on the interaction of drug colloidal carriers with the ocular mucosa. Eur J Pharm Sci. 2003;20:73-81. 
55. Law SL, Huang KJ, Chiang CH. Acyclovir containing liposomes for potential ocular delivery corneal penetration and absorption. J Control Release. 2000;63:135-40.

56. Attama AA, Reichl S, Muller-Goymann CC. Sustained release and permeation of timolol from surface modified solid lipid nanoparticles through bioengineered human cornea. Curr Eye Res. 2009;34:698-705.

57. Maurice DM, Riley MV. Ocular pharmacokinetics. In: Gragmore CN, editor. Biochemistry of the Eye. London, UK: Academic Press; 1970:6-16.
58. Loftsson T, Bodor N. The effect of cyclodextrins on percutaneous transport of drugs. In: Smith EW, Maibach HI, editors. Percutaneous Penetration Enhancers. Baton Rouge, FL: CRC Press; 1995:335-342.

59. Schoenwald RD, Huang HS. Corneal penetration behaviour of $\beta$-blocking agents I: Physicochemical factors. J Pharm Sci. 1983;72:1266-1272.

60. Coffin MD, McGinity JW. Biodegradable pseudolatexes: the chemical stability of poly(D,L-lactide) and poly( $\varepsilon$-caprolactone) nanoparticles in aqueous media. Pharm Res. 1992;9:200-205.
International Journal of Nanomedicine

\section{Publish your work in this journal}

The International Journal of Nanomedicine is an international, peerreviewed journal focusing on the application of nanotechnology in diagnostics, therapeutics, and drug delivery systems throughout the biomedical field. This journal is indexed on PubMed Central,

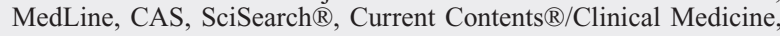

\section{Dovepress}

Journal Citation Reports/Science Edition, EMBase, Scopus and the Elsevier Bibliographic databases. The manuscript management system is completely online and includes a very quick and fair peer-review system, which is all easy to use. Visit http://www.dovepress.com/ testimonials.php to read real quotes from published authors.

Submit your manuscript here: http://www.dovepress.com/international-journal-of-nanomedicine-journal 